\title{
MINIMAX OPTIMAL CONTROL PROBLEMS. NUMERICAL ANALYSIS OF THE FINITE HORIZON CASE
}

\author{
Silvia C. Di Marco and Roberto L.V. González
}

\begin{abstract}
In this paper we consider the numerical computation of the optimal cost function associated to the problem that consists in finding the minimum of the maximum of a scalar functional on a trajectory. We present an approximation method for the numerical solution which employs both discretization on time and on spatial variables. In this way, we obtain a fully discrete problem that has unique solution. We give an optimal estimate for the error between the approximated solution and the optimal cost function of the original problem. Also, numerical examples are presented.

Résumé. Nous étudions ici la solution numérique d'une inéquation quasi-variationnelle associée à la minimisation du maximum d'une fonctionnelle définie sur la trajectoire d'un système dynamique gouverné par une équation différentielle ordinaire. Nous faisons la présentation d'une méthode d'approximation en employant des discrétisations en espace et en temps. Nous obtenons des estimations optimales pour la vélocité de convergence des solutions approchées vers la fonction de coût optimal du problème originel.
\end{abstract}

AMS Subject Classification. Primary: 49D25, 49A40, Secondary: 49C05, 49C20.

Received: November 19, 1996. Revised: June 16, 1998.

\section{INTRODUCTION AND DESCRIPTION OF THE PROBLEM}

\subsection{Description of the problem}

We consider in the interval $[0, T]$ a dynamic system which evolves according to the ordinary differential equation

$$
\mid \begin{aligned}
& \frac{d y}{d s}(s)=g(y(s), \alpha(s)) \quad 0 \leq t \leq s \leq T \\
& y(t)=x \in \mathbb{R}^{r} .
\end{aligned}
$$

The optimal control problem consists in minimizing the functional $J$

$$
\begin{aligned}
J:[0, T] \times \mathbb{R}^{r} \times \mathcal{A} & \mapsto \mathbb{R} \\
(t, x, \alpha(\cdot)) & \mapsto J(t, x, \alpha(\cdot))=\operatorname{ess} \sup \{f(y(s), \alpha(s)): s \in[t, T)\} .
\end{aligned}
$$

The set of admissible controls is given by $\mathcal{A}=L^{\infty}([0, T] ; A), A \subset \mathbb{R}^{m}$, and we also will use the notation:

$$
\mathcal{A}_{t}=L^{\infty}([t, T] ; A) \text {. }
$$

Key words and phrases. minimax problems, optimal cost function, discrete maximum principle, fully discrete solution. CONICET - Inst. Beppo Levi, Dpto. Matemática, FCEIA, Universidad Nacional de Rosario, Rosario, Argentine. e-mail: dimarco@fceia.unr.edu.ar, rlvgonza@fceia.unr.edu.ar 
The value function $u$ is defined by

$$
\begin{aligned}
u:[0, T] \times \mathbb{R}^{r} & \mapsto \mathbb{R} \\
(t, x) & \mapsto u(t, x)=\inf \left\{J(t, x, \alpha(\cdot)): \alpha(\cdot) \in \mathcal{A}_{t}\right\}
\end{aligned}
$$

The continuous problem arises, for example, when we want to minimize the maximum deviation of the controlled trajectories with respect to a given special trajectory. This differs from those problems usually considered in the optimal control literature, where a cumulative cost is minimized. As considering a cumulative cost is not always the best method to qualify the performance of a controlled system with a unique scalar parameter, problems of this type have received considerable interest in recent publications (see e.g. [2-10]).

Instead of being considered as an optimal control problem with a non-standard cost functional, this problem can also be studied through other different approaches, between them, the following two:

(a) It can be seen as an ordinary optimal control problem where the dynamic system evolves "very fast". In effect, introducing the auxiliary state variable $y_{r+1}$, which verifies the following differential inclusion (see [1]),

$$
\mid \begin{aligned}
& \frac{d y_{r+1}}{d s}(s) \in G\left(f(y(s), \alpha(s))-y_{r+1}(s)\right), \quad \text { a.e. } s \in(t, T), \\
& y_{r+1}(t)=0,
\end{aligned}
$$

where $G$ is given by

$$
G(v)= \begin{cases}0 & \text { if } v<0 \\ {[0, \infty]} & \text { if } v=0 \\ \infty & \text { if } v>0\end{cases}
$$

it is easy to check that $y_{r+1}(T)=\operatorname{ess} \sup \{f(y(s), \alpha(s)): s \in[t, T]\}$.

By considering the functional

$$
J_{T}(t, x, \alpha(\cdot))=y_{r+1}(T),
$$

we have an ordinary optimal control problem.

(b) The minimax problem can be analyzed as a disguised differential game problem.

In this game, one player tries to minimize the cost

$$
J(t, x, \alpha(\cdot), \tau)=f(y(\tau), \alpha(\tau))
$$

( $\tau$ denotes a stopping time of the process), while the opponent - using full information of the actions of the first player - chooses at any instant the stopping time $\tau$ of the process. As a result of the complete game, the pay-off (4) is given.

The objective of this work is to obtain a numerical approximation of the value function $u$ defined by (3).

Those interpretations of the original control problem (briefly discussed above), are non-standard and consequently, several numerical methods, as those presented in [11-13,19-22], cannot be directly used here. The numerical procedure presented in detailed form in this paper, was already announced in [16]. Our work comprises fundamentally two steps:

(i) We obtain a discrete time approximation using a finite differences scheme and we give an estimate of the error of this approximation. 
(ii) By using linear finite elements, we obtain a fully discrete approximation that converges to the solution of the original problem with rate $\sqrt{k}$. Besides, we show the optimality of the estimation $\sqrt{k}$.

Finally, we present some numerical applications with computational results.

\subsection{Technical assumptions and properties of the value function}

\section{Assumptions}

Let $B U C\left(\mathbb{R}^{r} \times A\right)$ be the set of bounded and uniformly continuous functions on $\mathbb{R}^{r} \times A$ and let $\operatorname{Lips}\left(\mathbb{R}^{r}\right)$ be the set of uniformly Lipschitz continuous functions on $\mathbb{R}^{r}$.

We assume that $f$ and $g$ satisfy the following hypotheses:

$\left(\mathrm{A}_{1}\right) g: \mathbb{R}^{r} \times A \mapsto \mathbb{R}^{r}, g \in B U C\left(\mathbb{R}^{r} \times A\right) ; g(\cdot, a) \in \operatorname{Lips}\left(\mathbb{R}^{r}\right), \forall a \in A$. The constants $M_{g}$ and $L_{g}$ satisfy, respectively

$$
\|g(x, a)\| \leq M_{g}, \quad\|g(x, a)-g(\widehat{x}, a)\| \leq L_{g}\|x-\widehat{x}\|, \quad \forall x, \widehat{x} \in \mathbb{R}^{r}, \forall a \in A
$$

$\left(\mathrm{A}_{2}\right) f: \mathbb{R}^{r} \times A \mapsto \mathbb{R}, f \in C\left(\mathbb{R}^{r} \times A\right) ; f(\cdot, a) \in \operatorname{Lips}\left(\mathbb{R}^{r}\right), \forall a \in A$. The constant $L_{f}$ satisfies

$$
|f(x, a)-f(\widehat{x}, a)| \leq L_{f}\|x-\widehat{x}\|, \quad \forall x, \widehat{x} \in \mathbb{R}^{r}, \forall a \in A .
$$

$\left(\mathrm{A}_{3}\right)$ The control set $A$ is compact in $\mathbb{R}^{\nu}$.

Properties of the value function

The following properties have been established by Barron-Ishii in [4] and [8]:

- The function $u$ is Lipschitz continuous in its spatial variable with Lipschitz constant $L_{u}$ $L_{u}=L_{f} \exp \left(L_{g} T\right)$.

- The value function $u$ satisfies the following dynamical programming principle $\forall t \in[0, T), x \in \mathbb{R}^{r}$

$$
\mid \begin{aligned}
& u(t, x)=\inf _{\alpha \in \mathcal{A}_{t}}\{\max \{u(s, y(s)), \underset{\tau \in[t, s]}{\operatorname{ess} \sup } f(y(\tau), \alpha(\tau))\}\}, \\
& u(T, x)=\min _{a \in A} f(x, a) .
\end{aligned}
$$

Remark 1.1. Similarly as to what was proved in [4], (if assumptions $\mathrm{A}_{1}$ and $\mathrm{A}_{2}$ hold), it results $u \in \operatorname{Lips}([0, T] \times$ $\left.\mathbb{R}^{r}\right)$. In other words, $u$ is Lipschitz continuous in both variables.

\section{A DisCRETE TIME SCHEME OF APPROXimation}

Here we introduce an auxiliary problem that is a natural discretization of the optimal cost $u$ defined in (3).

\subsection{The discrete time problem}

We divide the interval $[0, T]$ into $\mu$ sub-intervals with common length $h=T / \mu$. We define, for every $n=0, \ldots, \mu$

$$
\mathcal{A}_{n}^{h}=\left\{\alpha(\cdot) \in \mathcal{A}_{n h}: \alpha \text { constant in }[m h,(m+1) h), m=n, \ldots, \mu\right\} ;
$$


for each $\alpha^{h}(\cdot) \in \mathcal{A}_{n}^{h}$, the discrete time process $y^{h}$

$$
\mid \begin{aligned}
& y^{h}(m+1)=y^{h}(m)+h g\left(y^{h}(m), \alpha^{h}(m h)\right), \quad \forall m=n, \ldots, \mu-1, \\
& y^{h}(n)=x
\end{aligned}
$$

and the cost functional $J^{h}$

$$
J^{h}\left(n, x, \alpha^{h}(\cdot)\right)=\max _{m=n, \ldots, \mu} f\left(y^{h}(m), \alpha^{h}(m h)\right)
$$

The discrete time cost $u^{h}$ is given by

$$
u^{h}(n, x)=\min _{\alpha^{h} \in \mathcal{A}_{n}^{h}} J^{h}\left(n, x, \alpha^{h}(\cdot)\right) .
$$

Remark 2.1. The process $y^{h}$ is an Euler discretization of the continuous system (1). In (7) the minimum exists because $\mathcal{A}_{n}^{h}$ is compact in $A^{\mu-n}$ and $J^{h}$ is a continuous mapping from $\mathcal{A}_{n}^{h}$ to $\mathbb{R}$ by virtue of hypotheses $\mathrm{A}_{1}-\mathrm{A}_{3}$.

\subsection{Properties of the function $\mathbf{u}^{\mathrm{h}}$}

In the following proposition we establish the dynamic programming principle verified by the discrete time cost. It gives a recursive way to compute the function $u^{h}$. The proof follows classical lines and we omit it for the sake of brevity.

Proposition 2.1. For every $n=0, \ldots, \mu-1, u^{h}$ verifies the recursive relation

$$
u^{h}(n, x)=\min _{a \in A}\left\{\max \left\{f(x, a), u^{h}(n+1, x+h g(x, a))\right\}\right\},
$$

and the final condition

$$
u^{h}(\mu, x)=\min _{a \in A} f(x, a)
$$

It is easy to prove - using well known techniques as those that can be seen in [18] - that the function $u^{h}$ is Lipschitz continuous in its spatial variable with Lipschitz constant $L_{u}$. These results are established in the following proposition.

Proposition 2.2. For every $n=0, \ldots, \mu, x$ and $\widehat{x} \in \mathbb{R}^{r}$, it is valid that

$$
\left|u^{h}(n, x)-u^{h}(n, \widehat{x})\right| \leq L_{u}\|x-\widehat{x}\| .
$$

\subsection{Approximation of controls with step functions}

To compute the discrete time cost function $u^{h}$ defined in (7), we optimize the functional $J^{h}$ on the set $\mathcal{A}_{n}^{h}$, whose elements are step functions. To prove the convergence of $u^{h}$ to $u$, we need to establish some suitable relations between controls of $\mathcal{A}_{n h}$ and $\mathcal{A}_{n}^{h}$.

The relation $\mathcal{A}_{n}^{h} \subset \mathcal{A}_{n h}$ is obvious. To get results in the opposed sense, we will prove that given $\alpha(\cdot) \in \mathcal{A}_{n h}$ there exists $\alpha^{h}(\cdot) \in \mathcal{A}_{n}^{h}$ such that

$$
\lim _{h \rightarrow 0} g\left(y^{h}(\cdot), \alpha^{h}(\cdot)\right)=g(y(\cdot), \alpha(\cdot))
$$


in the weak- topology of $L^{\infty}\left([t, T] ; \mathbb{R}^{r}\right)$ and

$$
\liminf _{h \rightarrow 0} J^{h}\left(n, x, \alpha^{h}(\cdot)\right)=J(n h, x, \alpha(\cdot)) .
$$

To simplify the exposition, without losing generality, we focus on the particular case where the system in (1) starts in $t=0$.

For any $\mu_{1}$, we take $h_{1}=T / \mu_{1}$ and we divide the interval $[0, T]$ into $\mu_{1}$ sub-intervals $I_{i}$, where $I_{i}=\left[t_{i}, t_{i+1}\right)$, $t_{i}=i h_{1}$, and $i=0, \ldots, \mu_{1}-1$.

Remark 2.2. $L^{\infty}([0, T] ; A)$ can be divided into equivalence classes defined by the relation

$$
\alpha(\cdot) \sim \beta(\cdot) \text { iff } \alpha(t)=\beta(t), \text { a.e. } t \in[0, T] .
$$

It is clear that if $\alpha(\cdot)$ differs from $\beta(\cdot)$ in a zero measure set, so do $f(y(t), \alpha(t))$ and $f(y(t), \beta(t))$. Therefore, at each sub-interval $I_{i}$, it is possible to choose $\alpha(\cdot)$ (an element of the equivalence class) such that

$$
\operatorname{ess} \sup \left\{f(y(\cdot), \alpha(\cdot)): t \in I_{i}\right\}=\sup \left\{f(y(\cdot), \alpha(\cdot)): t \in I_{i}\right\} .
$$

From now, we consider a control $\alpha(\cdot) \in L^{\infty}([0, T] ; A)$ that verifies the property (10) for every $I_{i}$.

Definition 2.1. Given $\alpha(\cdot) \in \mathcal{A}_{0}$, we define $A(\alpha, i):=\left\{\alpha(s): s \in I_{i}\right\}$ and $\Gamma(\alpha, i):=\overline{A(\alpha, i)}$ its closure. Clearly, by hypothesis $\mathrm{A}_{3}, \Gamma(\alpha, i)$ is compact.

Lemma 2.1. Let $r$ be the dimension of the state space. For every $i=0, \ldots, \mu_{1}-1$, there exists a step function $\alpha_{w}: I_{i} \mapsto \Gamma(\alpha, i)$ that takes at most $r+1$ constant values, such that

$$
\begin{gathered}
\int_{t_{i}}^{t_{i+1}} g\left(y\left(t_{i}\right), \alpha_{w}(s)\right) d s=\int_{t_{i}}^{t_{i+1}} g\left(y\left(t_{i}\right), \alpha(s)\right) d s, \\
\max _{s \in I_{i}} f\left(y\left(t_{i}\right), \alpha_{w}(s)\right) \leq \sup _{s \in I_{i}} f\left(y\left(t_{i}\right), \alpha(s)\right) .
\end{gathered}
$$

Proof. Since $g$ is continuous and $\Gamma(\alpha, i)$ is compact we have that $g\left(y\left(t_{i}\right), \Gamma(\alpha, i)\right)$ is also compact and then, from the Convex Analysis Theory it follows that $\operatorname{Co}\left(g\left(y\left(t_{i}\right), \Gamma(\alpha, i)\right)\right)$ is closed, i.e.

$$
\overline{\operatorname{Co}\left(g\left(y\left(t_{i}\right), \Gamma(\alpha, i)\right)\right)}=\operatorname{Co}\left(g\left(y\left(t_{i}\right), \Gamma(\alpha, i)\right)\right) .
$$

It is clear that

$$
g_{i}=\frac{1}{h_{1}} \int_{t_{i}}^{t_{i+1}} g\left(y\left(t_{i}\right), \alpha(s)\right) d s \in \overline{C o\left(g\left(y\left(t_{i}\right), \Gamma(\alpha, i)\right)\right)}
$$

and then

$$
g_{i} \in \operatorname{Co}\left(g\left(y\left(t_{i}\right), \Gamma(\alpha, i)\right)\right)
$$

Being $r$ the dimension of the state set, from the Caratheodory Theorem (see [15], p. 42) it follows that there exists $\left\{a_{k i}: k=1, \ldots, r+1\right\}$, and $\left\{\lambda_{k i}: k=1, \ldots, r+1\right\}$, with $\sum_{k=1}^{r+1} \lambda_{k i}=1, \lambda_{k i} \geq 0$ such that

$$
g_{i}=\frac{1}{h_{1}} \sum_{k=1}^{r+1} \lambda_{k i} g\left(y\left(t_{i}\right), a_{k i}\right)
$$


We consider a partition of $I_{i},\left\{I_{k i}: k=1, \ldots, r+1\right\}$ such that $\left|I_{k i}\right|=h_{1} \lambda_{k i}$ and we define $\alpha_{w}(t)=a_{k i}$, for every $t \in I_{k i}$.

It is clear that $\alpha_{w}$ takes at most $r+1$ constant values in $\Gamma(\alpha, i)$ and we have

$$
\int_{t_{i}}^{t_{i+1}} g\left(y\left(t_{i}\right), \alpha_{w}(s)\right) d s=\int_{t_{i}}^{t_{i+1}} g\left(y\left(t_{i}\right), \alpha(s)\right) d s .
$$

To prove (12), let us note that if $s \in I_{i}$, then $\alpha_{w}(s) \in \Gamma(\alpha, i)$. Therefore,

$$
\begin{aligned}
\max \{ & \left.f\left(y\left(t_{i}\right), \alpha_{w}(s)\right): s \in I_{i}\right\} \\
& \leq \max \left\{f\left(y\left(t_{i}\right), a\right): a \in \Gamma(\alpha, i)\right\}=\sup \left\{f\left(y\left(t_{i}\right), a\right): a \in A(\alpha, i)\right\} .
\end{aligned}
$$

The following lemma gives an estimate for the difference between the original trajectory of the system and the trajectory corresponding to the step control $\alpha_{w}(\cdot)$.

Lemma 2.2. Let $y(\cdot)$ be the response to the control $\alpha(\cdot)$ and $y_{w}(\cdot)$, the response to the control $\alpha_{w}(\cdot)$, then

$$
\left\|y(t)-y_{w}(t)\right\| \leq M h_{1}
$$

where

$$
M=\left(2+\exp \left(L_{g} t\right)\right) M_{g} .
$$

Proof. We define, $\forall i=0, \ldots, \mu-1, E_{i}=\left\|y\left(t_{i}\right)-y_{w}\left(t_{i}\right)\right\|$. In consequence, it is valid that

$$
E_{i+1} \leq E_{i}+\left\|\int_{t_{i}}^{t_{i+1}}\left(g(y(s), \alpha(s))-g\left(y_{w}(s), \alpha_{w}(s)\right)\right) d s\right\| .
$$

To estimate the second term of (15), we write

$$
\begin{aligned}
& \left\|\int_{t_{i}}^{t_{i+1}}\left(g(y(s), \alpha(s))-g\left(y_{w}(s), \alpha_{w}(s)\right)\right) d s\right\| \\
& \leq\left\|\int_{t_{i}}^{t_{i+1}}\left(g(y(s), \alpha(s))-g\left(y\left(t_{i}\right), \alpha(s)\right)\right) d s\right\| \\
& +\left\|\int_{t_{i}}^{t_{i+1}}\left(g\left(y\left(t_{i}\right), \alpha(s)\right)-g\left(y\left(t_{i}\right), \alpha_{w}(s)\right)\right) d s\right\| \\
& +\left\|\int_{t_{i}}^{t_{i+1}}\left(g\left(y\left(t_{i}\right), \alpha_{w}(s)\right)-g\left(y_{w}\left(t_{i}\right), \alpha_{w}(s)\right)\right) d s\right\| \\
& +\left\|\int_{t_{i}}^{t_{i+1}}\left(g\left(y_{w}\left(t_{i}\right), \alpha_{w}(s)\right)-g\left(y_{w}(s), \alpha_{w}(s)\right)\right) d s\right\| .
\end{aligned}
$$


From (1) and $\left(\mathrm{A}_{1}\right)$, we have

$$
\left\|y(s)-y\left(t_{i}\right)\right\|=\left\|\int_{t_{i}}^{s} g(y(\theta), \alpha(\theta)) d \theta\right\| \leq M_{g}\left(s-t_{i}\right) .
$$

Then, using $\left(\mathrm{A}_{1}\right)$ and (17), we estimate the first term of (16) in the following way:

$$
\begin{aligned}
& \left\|\int_{t_{i}}^{t_{i+1}}\left(g(y(s), \alpha(s))-g\left(y\left(t_{i}\right), \alpha(s)\right)\right) d s\right\| \\
& \quad \leq \int_{t_{i}}^{t_{i+1}}\left\|g(y(s), \alpha(s))-g\left(y\left(t_{i}\right), \alpha(s)\right)\right\| d s \\
& \leq L_{g} \int_{t_{i}}^{t_{i+1}}\left\|y(s)-y\left(t_{i}\right)\right\| d s \leq L_{g} M_{g} \int_{t_{i}}^{t_{i+1}}\left(s-t_{i}\right) d s \leq L_{g} M_{g} \frac{h_{1}^{2}}{2} .
\end{aligned}
$$

We can estimate in a similar way the fourth term of (16),

$$
\left\|\int_{t_{i}}^{t_{i+1}}\left(g\left(y_{w}\left(t_{i}\right), \alpha_{w}(s)\right)-g\left(y_{w}(s), \alpha_{w}(s)\right)\right) d s\right\| \leq L_{g} M_{g} \frac{h_{1}^{2}}{2} .
$$

From (11), the second term of (16) is zero, i.e.

$$
\left\|\int_{t_{i}}^{t_{i+1}}\left(g\left(y\left(t_{i}\right), \alpha(s)\right)-g\left(y\left(t_{i}\right), \alpha_{w}(s)\right)\right) d s\right\|=0 .
$$

From $\left(\mathrm{A}_{1}\right)$, the third term of $(16)$ can be estimated as follows,

$$
\begin{aligned}
& \left\|\int_{t_{i}}^{t_{i+1}}\left(g\left(y\left(t_{i}\right), \alpha_{w}(s)\right)-g\left(y_{w}\left(t_{i}\right), \alpha_{w}(s)\right)\right)\right\| \\
& \leq \int_{t_{i}}^{t_{i+1}}\left\|g\left(y\left(t_{i}\right), \alpha_{w}(s)\right)-g\left(y_{w}\left(t_{i}\right), \alpha_{w}(s)\right)\right\| d s \\
& \leq L_{g} \int_{t_{i}}^{t_{i+1}}\left\|y\left(t_{i}\right)-y_{w}\left(t_{i}\right)\right\| d s \leq E_{i} L_{g} h_{1} .
\end{aligned}
$$

From $(15,16,18-21)$, we get

$$
E_{i+1} \leq E_{i}\left(1+L_{g} h_{1}\right)+L_{g} M_{g} h_{1}^{2}
$$


Taking into account that $E_{o}=0$, by induction we can prove that

$$
E_{i} \leq L_{g} M_{g} h_{1}^{2} \sum_{j=0}^{i-1}\left(1+L_{g} h_{1}\right)^{j}
$$

Since $\sum_{j=0}^{i-1}\left(1+L_{g} h_{1}\right)^{j}=\frac{\left(1+L_{g} h_{1}\right)^{i}-1}{\left(1+L_{g} h_{1}\right)-1}$, we obtain $E_{i} \leq M_{g} h_{1}\left(1+L_{g} h_{1}\right)^{i}$.

Let $t \in I_{i}$, for some $i=0, \ldots, \mu_{1}-1$, we have

$$
\left\|y(t)-y_{w}(t)\right\| \leq\left\|y\left(t_{i}\right)-y_{w}\left(t_{i}\right)\right\|+\left\|\int_{t_{i}}^{t}\left(g(y(s), \alpha(s))-g\left(y_{w}(s), \alpha_{w}(s)\right)\right) d s\right\| .
$$

To estimate $(22)$, from $\left(A_{1}\right)$ we have

$$
\left\|\int_{t_{i}}^{t}\left(g(y(s), \alpha(s))-g\left(y_{w}(s), \alpha_{w}(s)\right)\right) d s\right\| \leq 2 M_{g} h_{1}
$$

and thus, we obtain

$$
\left\|y(t)-y_{w}(t)\right\| \leq 2 M_{g} h_{1}+M_{g} h_{1}\left(1+L_{g} h_{1}\right)^{i} \leq M_{g} h_{1}\left(2+\exp \left(L_{g} t\right)\right) .
$$

\subsection{Approximation of controls with uniform-step functions}

The control $\alpha_{w}$ is a step function which has, at most, $r+1$ steps in each interval of length $h_{1}$ (where $r$ is the dimension of the state space). We are interested in approximating the set $\mathcal{A}_{0}$ with uniform-step functions, so we need a suitable element of $\mathcal{A}_{0}^{h}$ that approximates $\alpha_{w}$ in the sense described below.

Construction of the control $\alpha_{w}^{h}$

The symbol $[s]$ denotes the integer part of $s$. For any interval $I_{i}=\left[t_{i}, t_{i+1}\right), t_{i}=i h_{1}$, we know that $\alpha_{w}$ takes, at most, $r+1$ different values denoted by $\alpha_{i j}, i=0, \ldots, \mu-1, j=0, \ldots, r$. Besides, $\lambda_{i j}$ denotes the length of the sub-interval where $\alpha_{w}=\alpha_{i j}$.

Given $\nu$ we define $h=\frac{h_{1}}{[\nu]}$,

$$
\mid \begin{array}{ll}
t_{i o}=t_{i} & i=0, \ldots, \mu_{1}-1, \\
t_{i j}=t_{i}+\sum_{k=0}^{j-1} \lambda_{i k} & j=1, \ldots, r+1, \\
\tilde{t}_{i j}=h\left[t_{i j} / h\right] & i=0, \ldots, \mu_{1}-1, j=0, \ldots, r+1 .
\end{array}
$$

We define $\forall s \in\left[\widetilde{t}_{i j}, \widetilde{t}_{i, j+1}\right)$

$$
\alpha_{w}^{h}(s)=\alpha_{i j}
$$


Therefore, $\alpha_{w}^{h}=\alpha_{w}$ in $I_{i}$, except at most in $r+1$ intervals of length $\eta_{i j}$, with $\eta_{i j}=t_{i j}-\widetilde{t}_{i j} \leq h$. Then, $\alpha_{w}^{h}=\alpha_{w}$ in $I_{i}$ except in a set which measure is smaller than

$$
\sum_{j=1}^{r+1} \eta_{i j} \leq(r+1) h
$$

Properties of the control $\alpha_{w}^{h}$

The procedure of construction of $\alpha_{w}^{h}$ implies that

$$
\mid \begin{aligned}
& \alpha_{w}^{h} \in \mathcal{A}_{0}^{h}, \\
& \left\{\alpha_{w}^{h}(s): s \in I_{i}\right\}=\left\{\alpha_{w}(s): s \in I_{i}\right\}, \forall i=0, \ldots, \mu_{1}-1 .
\end{aligned}
$$

By these properties, it is valid that

$$
\max _{s \in I_{i}} f\left(y\left(t_{i}\right), \alpha_{w}^{h}(s)\right)=\max _{s \in I_{i}} f\left(y\left(t_{i}\right), \alpha_{w}(s)\right) .
$$

From (12) and (25), we have

$$
\max _{s \in I_{i}} f\left(y\left(t_{i}\right), \alpha_{w}^{h}(s)\right) \leq \sup _{s \in I_{i}} f\left(y\left(t_{i}\right), \alpha(s)\right)
$$

Remark 2.3. In the following proofs we suppose, without losing generality, that $h \leq 1$.

The following lemma gives an estimate for the difference between the original trajectory of the system and the response corresponding to the uniform-step control $\alpha_{w}^{h}(\cdot)$.

Lemma 2.3. Let $y(\cdot)$ be the response to the control $\alpha(\cdot)$ and $y_{w}^{h}(\cdot)$ the response to the control $\alpha_{w}^{h}(\cdot)$, then

$$
\left\|y(t)-y_{w}^{h}(t)\right\| \leq K \sqrt{h}
$$

where

$$
K=\left(4+3 \exp \left(L_{g} t\right)\right) M_{g} \sqrt{\frac{2(r+1)}{L_{g}}} .
$$

Proof. To estimate the difference between $y(\cdot)$ and $y_{w}^{h}(\cdot)$, we write

$$
\left\|y(t)-y_{w}^{h}(t)\right\| \leq\left\|y(t)-y_{w}(t)\right\|+\left\|y_{w}(t)-y_{w}^{h}(t)\right\| .
$$

The first term of (27) was analyzed in Lemma 2.2 and a bound was given in (13). We will estimate now the second term of (27). Let $t \in I_{i}$, for some $i=0, \ldots, \mu_{1}-1$

$$
\begin{aligned}
\left\|y_{w}(t)-y_{w}^{h}(t)\right\| \leq & \left\|y_{w}\left(t_{i}\right)-y_{w}^{h}\left(t_{i}\right)\right\| \\
& +\left\|\int_{t_{i}}^{t}\left(g\left(y_{w}(s), \alpha_{w}(s)\right)-g\left(y_{w}^{h}(s), \alpha_{w}^{h}(s)\right)\right) d s\right\| .
\end{aligned}
$$

The second term of (28) is bounded by 


$$
\left\|\int_{t_{i}}^{t}\left(g\left(y_{w}(s), \alpha_{w}(s)\right)-g\left(y_{w}^{h}(s), \alpha_{w}^{h}(s)\right)\right) d s\right\| \leq 2 M_{g} h_{1} .
$$

To estimate the first one, we define $\forall i=0, \ldots, \mu_{1}-1$

$$
E_{i}=\left\|y_{w}\left(t_{i}\right)-y_{w}^{h}\left(t_{i}\right)\right\|
$$

then

$$
E_{i+1} \leq E_{i}+\left\|\int_{t_{i}}^{t_{i+1}}\left(g\left(y_{w}\left(t_{i}\right), \alpha_{w}(s)\right)-g\left(y_{w}^{h}\left(t_{i}\right), \alpha_{w}^{h}(s)\right)\right) d s\right\| .
$$

To estimate the second term of the right side of (31), we write

$$
\begin{aligned}
& g\left(y_{w}(s), \alpha_{w}(s)\right)-g\left(y_{w}^{h}(s), \alpha_{w}^{h}(s)\right)= \\
& =\quad\left(g\left(y_{w}(s), \alpha_{w}(s)\right)-g\left(y_{w}\left(t_{i}\right), \alpha_{w}(s)\right)\right)+\left(g\left(y_{w}\left(t_{i}\right), \alpha_{w}(s)\right)-g\left(y_{w}^{h}\left(t_{i}\right), \alpha_{w}(s)\right)\right) \\
& \quad+\left(g\left(y_{w}^{h}\left(t_{i}\right), \alpha_{w}(s)\right)-g\left(y_{w}^{h}\left(t_{i}\right), \alpha_{w}^{h}(s)\right)\right)+\left(g\left(y_{w}^{h}\left(t_{i}\right), \alpha_{w}^{h}(s)\right)-g\left(y_{w}^{h}(s), \alpha_{w}^{h}(s)\right)\right) .
\end{aligned}
$$

It is clear that the following estimates hold:

$$
\begin{aligned}
& \left\|\int_{t_{i}}^{t_{i+1}}\left(g\left(y_{w}(s), \alpha_{w}(s)\right)-g\left(y_{w}\left(t_{i}\right), \alpha_{w}(s)\right)\right) d s\right\| \leq L_{g} M_{g} \frac{h_{1}^{2}}{2}, \\
& \left\|\int_{t_{i}}^{t_{i+1}}\left(g\left(y_{w}^{h}\left(t_{i}\right), \alpha_{w}^{h}(s)\right)-g\left(y_{w}^{h}(s), \alpha_{w}^{h}(s)\right)\right) d s\right\| \leq L_{g} M_{g} \frac{h_{1}^{2}}{2}, \\
& \left\|\int_{t_{i}}^{t_{i+1}}\left(g\left(y_{w}\left(t_{i}\right), \alpha_{w}(s)\right)-g\left(y_{w}^{h}\left(t_{i}\right), \alpha_{w}(s)\right)\right) d s\right\| \leq L_{g} E_{i} h_{1} .
\end{aligned}
$$

From (24), it results that

$$
\left\|\int_{t_{i}}^{t_{i+1}}\left(g\left(y_{w}^{h}\left(t_{i}\right), \alpha_{w}(s)\right)-g\left(y_{w}^{h}\left(t_{i}\right), \alpha_{w}^{h}(s)\right)\right) d s\right\| \leq 2 M_{g}(r+1) h .
$$

In consequence, by substituting (32-35) into (31), we have 


$$
E_{i+1} \leq E_{i}\left(1+L_{g} h_{1}\right)+2 M_{g}(r+1) h+L_{g} M_{g} h_{1}^{2} .
$$

By induction, and taking into account (30), we obtain

$$
E_{i} \leq\left(2 M_{g}(r+1) h+L_{g} M_{g} h_{1}^{2}\right) \sum_{j=0}^{i-1}\left(1+L_{g} h_{1}\right)^{j} .
$$

Since $\sum_{j=0}^{i-1}\left(1+L_{g} h_{1}\right)^{j}=\frac{\left(1+L_{g} h_{1}\right)^{i}-1}{L_{g} h_{1}}$ and $\left(1+L_{g} h_{1}\right)^{i} \leq \exp \left(L_{g} t\right)$, we obtain the estimate

$$
E_{i} \leq\left(2 M_{g}(r+1) h+L_{g} M_{g} h_{1}^{2}\right) \frac{\exp \left(L_{g} t\right)}{L_{g} h_{1}} .
$$

The minimum of the right side of (36) is achieved at

$$
\nu=\sqrt{\frac{2(r+1)}{h L_{g}}} .
$$

We suppose, for the sake of simplicity, that $\nu$ is an integer (the general case can be proved similarly with no difficulty). Consequently, from (36) and (37), it results

$$
E_{i} \leq 2 \exp \left(L_{g} t\right) M_{g} \sqrt{\frac{2(r+1) h}{L_{g}}} .
$$

The inequality (28) is bounded by

$$
\left\|y_{w}(t)-y_{w}^{h}(t)\right\| \leq 2 M_{g} h_{1}+2 \exp \left(L_{g} t\right) M_{g} \sqrt{\frac{2(r+1)}{L_{g}}} \sqrt{h} .
$$

Finally, from (13), (14) and (38) we have the estimate

$$
\left\|y(t)-y_{w}^{h}(t)\right\| \leq K \sqrt{h}
$$

where $K=\left(4+3 \exp \left(L_{g} t\right)\right) M_{g} \sqrt{\frac{2(r+1)}{L_{g}}}$.

\subsection{Rate of convergence}

In the previous section, for each policy $\alpha \in \mathcal{A}_{0}$ and its associated response $y(\cdot)$, we have defined an approximating control $\alpha_{w}^{h} \in \mathcal{A}_{0}^{h}$ and we have obtained an estimate for the difference between the corresponding trajectories. In this section, we will give an estimate of the difference between $u^{h}$ and $u$. To do that, we define, for each $n=0, \ldots, \mu$, the following auxiliary function, which is the optimal cost evaluated on the uniform-step control functions 


$$
u^{e}(n, x)=\min _{\alpha^{h} \in \mathcal{A}_{n}^{h}} J\left(n h, x, \alpha^{h}(\cdot)\right)
$$

Lemma 2.4. Let $u^{e}(0, x)$ and $u^{h}(0, x)$ be the optimal cost defined in (7) and the discrete time cost defined in (7) respectively. Then

$$
\left|u^{e}(0, x)-u^{h}(0, x)\right| \leq C h .
$$

Proof. This result is easily obtained taking into account that the error associated to Euler's integration method applied to the system (1) is of order $h$. In effect, let $\left\{I_{i}: i=1, \ldots, \mu-1\right\}$ be a partition of $[0, T]$ of length $h$ with $I_{i}=\left[t_{i}, t_{i+1}\right]$ and let $t \in I_{i}$. Let $y^{h}(\cdot)$ be the function defined in (5) and $y_{\alpha^{h}}(\cdot)$ be the response to the uniform step control $\alpha^{h}(\cdot)$. With arguments similar to those used in the proof corresponding to Lemma 2.2, it can be obtained that

$$
\left\|y^{h}(i)-y_{\alpha^{h}}\left(t_{i}\right)\right\| \leq \frac{M_{g} L_{g} T}{2} h+O\left(h^{2}\right)
$$

From (1) it is evident that

$$
\left\|y_{\alpha^{h}}(t)-y_{\alpha^{h}}\left(t_{i}\right)\right\| \leq M_{g} h,
$$

in consequence, there exists $M$ (independent on the parameter $h$ ) such that

$$
\left|f\left(y^{h}(i), \alpha^{h}(t)\right)-f\left(y_{\alpha^{h}}(t), \alpha^{h}(t)\right)\right| \leq L_{f} M h .
$$

Since

$$
\begin{gathered}
J^{h}\left(0, x, \alpha^{h}(\cdot)\right)=\max _{i=0, \ldots, \mu-1} f\left(y\left(t_{i}\right), \alpha^{h}(s)\right), \\
J\left(0, x, \alpha^{h}(\cdot)\right)=\max _{i=0, \ldots, \mu-1}\left\{\sup _{s \in I_{i}} f\left(y_{\alpha^{h}}(s), \alpha(s)\right)\right\},
\end{gathered}
$$

it easy to see that

$$
\left|J^{h}\left(0, x, \alpha^{h}(\cdot)\right)-J\left(0, x, \alpha^{h}(\cdot)\right)\right| \leq L_{f} M h .
$$

Then,

$$
\left|u^{e}(0, x)-u^{h}(0, x)\right| \leq C h .
$$

Note 2.1. To simplify the notation and the exposition, $C$ and $M$ denote any generic constant whose value depends on the context where it appears. Such constants only depend on the functions $f$ and $g$ of the problem but they are independent on the parameters $h, k, \rho$ of discretization.

Theorem 2.1. Let $u(0, x)$ be the optimal cost of the original problem and $u^{h}(0, x)$ the discrete time cost defined in (7), then

$$
\left|u(0, x)-u^{h}(0, x)\right| \leq M \sqrt{h}
$$


Proof. Using the auxiliary definition (39) we can write:

$$
\left|u(0, x)-u^{h}(0, x)\right| \leq\left|u(0, x)-u^{e}(0, x)\right|+\left|u^{e}(0, x)-u^{h}(0, x)\right| .
$$

Let us now estimate the first term of the right side of (42). Since $\mathcal{A}_{0}^{h} \subset \mathcal{A}_{0}$, we have by definition (39)

$$
u(0, x) \leq u^{e}(0, x) .
$$

On the other hand, let us consider $\alpha \in \mathcal{A}_{0}$ and $\alpha_{w}^{h} \in \mathcal{A}_{0}^{h}$ the control defined in (23) (which is the uniform step control function associated to $\alpha$ ). Then we have

$$
J\left(0, x, \alpha_{w}^{h}\right)=\sup _{s \in[0, T)} f\left(y_{w}^{h}(s), \alpha_{w}^{h}(s)\right) .
$$

Let $s \in I_{i}$,

$$
\begin{aligned}
f\left(y_{w}^{h}(s), \alpha_{w}^{h}(s)\right)= & \left(f\left(y_{w}^{h}(s), \alpha_{w}^{h}(s)\right)-f\left(y(s), \alpha_{w}^{h}(s)\right)\right) \\
& +\left(f\left(y(s), \alpha_{w}^{h}(s)\right)-f\left(y\left(t_{i}\right), \alpha_{w}^{h}(s)\right)\right)+f\left(y\left(t_{i}\right), \alpha_{w}^{h}(s)\right),
\end{aligned}
$$

by $\left(\mathrm{A}_{2}\right)$ and Lemma 2.3, we have

$$
\begin{gathered}
\left|f\left(y_{w}^{h}(s), \alpha_{w}^{h}(s)\right)-f\left(y(s), \alpha_{w}^{h}(s)\right)\right| \leq C \sqrt{h}, \\
\left|f\left(y(s), \alpha_{w}^{h}(s)\right)-f\left(y\left(t_{i}\right), \alpha_{w}^{h}(s)\right)\right| \leq C h .
\end{gathered}
$$

From (26), we obtain

$$
\begin{aligned}
\sup _{s \in[0, T)} f\left(y\left(t_{i}\right), \alpha_{w}^{h}(s)\right) & =\max _{i=0, \ldots, \mu_{1}-1}\left\{\max _{s \in I_{i}} f\left(y\left(t_{i}\right), \alpha_{w}^{h}(s)\right)\right\} \\
& \leq \max _{i=0, \ldots, \mu_{1}-1}\left\{\sup _{s \in I_{i}} f\left(y\left(t_{i}\right), \alpha(s)\right)\right\} \leq J(0, x, \alpha(\cdot))+L_{f} M_{g} h .
\end{aligned}
$$

Consequently, from $(2,44,45)$, we get

$$
J\left(0, x, \alpha_{w}^{h}(\cdot)\right) \leq J(0, x, \alpha(\cdot))+M \sqrt{h},
$$

therefore

$$
u^{e}(0, x) \leq J\left(0, x, \alpha_{w}^{h}(\cdot)\right) \leq J(0, x, \alpha(\cdot))+M \sqrt{h} .
$$

Taking the infimum over $\mathcal{A}_{0}$ in the right side of (46), we have

$$
u^{e}(0, x) \leq u(0, x)+M \sqrt{h} .
$$

From $(43,47)$, we get

$$
\left|u^{e}(0, x)-u(0, x)\right| \leq M \sqrt{h} .
$$


Finally, from $(40,48)$, we obtain

$$
\left|u(0, x)-u^{h}(0, x)\right| \leq M \sqrt{h}
$$

Remark 2.4. In a similar way as it was done in Theorem $2.1, \forall n=0, \ldots, \mu$, we can also prove that the following inequality holds

$$
\left|u(n, x)-u^{h}(n, x)\right| \leq M \sqrt{h} .
$$

\section{Fully Discrete solutions}

In the previous section we have approximated the function $u$ with one obtained by discretizing the original problem in its time variable. This approximation scheme is not directly implementable to be computed numerically. To obtain a fully discrete approximation with this property, we discretize the space $\mathbb{R}^{r}$, using the methodology described in $[21,22]$.

\subsection{Elements of the discrete problem}

We identify the discretization of the spatial variables with the parameter $k$, which also indicates the size of the discretization. The symbols $X^{\circ}$ and $\operatorname{diam}(X)$ denote respectively the interior and the diameter of a set $X$.

\section{Approximation of the domain $\mathbb{R}^{r}$}

We consider a family of quasi-uniform triangulations of $\mathbb{R}^{r}$, which is denoted by $\left\{\mathcal{S}^{k}\right\}_{k}$ and verifies:

- For all $k, \mathcal{S}^{k}$ is a denumerable collection of closed simplices $\left\{S_{j}^{k}\right\}_{j}$ such that $\bigcup_{j} S_{j}^{k}=\mathbb{R}^{r}$.

- If $S_{j}^{k} \in \mathcal{S}^{k}, S_{p}^{k} \in \mathcal{S}^{k}, S_{j}^{k} \neq S_{p}^{k}$, we have

$-\left(S_{j}^{k}\right)^{\circ} \bigcap\left(S_{p}^{k}\right)^{\circ}=\emptyset$.

- Either $S_{j}^{k} \bigcap S_{p}^{k}=\emptyset$ or $S_{j}^{k}$ and $S_{p}^{k}$ have in common a whole $(r-m)$ - edge, $m=1, \ldots, r$.

- $\max _{j}\left(\operatorname{diam}\left(S_{j}^{k}\right)\right)=k$,

- $\exists \chi_{1}^{j}>0$ and $\exists \chi_{2}>0$ independent on the discretization, such that, denoting by $d_{j}$ the diameter of the simplex $S_{j}^{k}$, it is verified

- the simplex $S_{j}^{k}$ has a sphere of radius $r_{j}$ in its interior and it results $r_{j} \geq \chi_{1} d_{j}$,

- for any simplex $S_{j}^{k}, k \leq \chi_{2} d_{j}$.

Let $V_{k}=\left\{x^{i}, i \in \mathbb{N}\right\}$ be the vertices of $\bigcup_{j} S_{j}^{k}$, arbitrarily arranged. Every $x \in \mathbb{R}^{r}$ is a convex combination of the vertices $x^{i}$ of the simplex to which $x$ belongs. Hence, $\forall a \in A$ there exists a matrix with components $\gamma_{j}\left(x^{i}, a\right)$, such that for each $i \in \mathbb{N}$ :

$$
\mid \begin{array}{ll}
\gamma_{j}\left(x^{i}, a\right) \geq 0, & \forall j \in \mathbb{N}, \\
\gamma_{j}\left(x^{i}, a\right)>0, & \text { for at most }(\mathrm{r}+1) \text { values of } j, \\
\sum_{j=1}^{\infty} \gamma_{j}\left(x^{i}, a\right)=1, & \\
x^{i}+h g\left(x^{i}, a\right)=\sum_{j=1}^{\infty} \gamma_{j}\left(x^{i}, a\right) x^{j} . &
\end{array}
$$


Definition of the approximate space $F_{k}$

We consider the set $W_{k}$ of functions $w: \mathbb{R}^{r} \mapsto \mathbb{R}, w \in W^{1, \infty}\left(\mathbb{R}^{r}\right)$, such that $\partial w / \partial x$ is constant in the interior of each simplex, i.e., the functions $w$ are linear finite elements and they are characterized by their values on $V_{k}$. We denote

$$
F_{k}=\left(W_{k}\right)^{\mu+1}
$$

The elements of $F_{k}$ will be denoted by $w_{k}^{h}(n, x), n=0, \ldots, \mu, x \in V_{k}$.

Definition of the fully discrete solution

Taking in mind the equations (8)-(9), we define the fully discrete solution to be the function $u_{k}^{h} \in F_{k}$ which verifies the following recurrence $\forall x^{i} \in V_{k}$,

$$
\begin{array}{ll}
u_{k}^{h}\left(n, x^{i}\right)=\min _{a \in A}\left\{\max \left\{f\left(x^{i}, a\right), \sum_{j=1}^{\infty} \gamma_{j}\left(x^{i}, a\right) u_{k}^{h}\left(n+1, x^{j}\right)\right\}\right\}, & \\
u_{k}^{h}\left(\mu, x^{i}\right)=\min _{a \in A} f\left(x^{i}, a\right) . & \forall n=0, \ldots, \mu-1,
\end{array}
$$

Remark 3.1. Obviously, the solution of (50) is unique and can be computed recursively. This allows us to implement the computational procedure.

Remark 3.2. If $A$ is an infinite set, then (50) is not a fully discrete scheme in a strict sense. To obtain a scheme of that sort, we should perform a final step of discretization which consists in the approximation of the compact set $A$ by a suitable finite one. In order to simplify the exposition, in this paper we have omitted such

approximation and so, in the following we will suppose that the operation $\min _{a \in A}\{\ldots\}$ can be computed exactly in the numerical implementation of (50).

\subsection{Central result}

\subsubsection{Rate of convergence}

To obtain an estimate of the rate of convergence of the fully discrete solution, we establish an auxiliary result given by the following theorem, which brings an estimate for the difference between the discrete time cost and the fully discrete cost (defined in (8) and (50), respectively). The proof is based on regularization techniques and the obtained estimate depends on the regularization parameter $\rho$.

Theorem 3.1. Let $x \in V_{k}$ and $n=0, \ldots, \mu$, then it results

$$
\left|u^{h}(n, x)-u_{k}^{h}(n, x)\right| \leq M \rho+T\left(L_{u} L_{g} \rho+L_{u} \frac{k^{2}}{\rho h}\right) .
$$

To prove this theorem, we apply properties established in subsection 3.2.2. The proof (given in Sect. 3.2.3) consists in obtaining estimates for the differences between subsolutions and supersolutions of problems introduced ad-hoc.

The central result is given by the following theorem which establishes an estimate of the difference between the optimal cost and the fully discrete solution.

Theorem 3.2. If there exists constants $c_{1}$ and $c_{2}$ such that $c_{1} k \leq h \leq c_{2} k$, then there exists a constant $C$ such that $\forall x \in V_{k}$ and $\forall n=0, \ldots, \mu$, it results

$$
\left|u(n h, x)-u_{k}^{h}(n, x)\right| \leq C \sqrt{k} .
$$


Proof. $\quad$ By using both the results of theorems 2.1 and 3.1, we conclude that

$$
\left|u(n h, x)-u_{k}^{h}(n, x)\right| \leq C \sqrt{h}+M \rho+T\left(L_{u} L_{g} \rho+L_{u} \frac{k^{2}}{\rho h}\right) .
$$

The estimate obtained in (52) attains its minimum value at $\rho=C k / \sqrt{h}$, in consequence

$$
\left|u(n h, x)-u_{k}^{h}(n, x)\right| \leq C\left(\sqrt{h}+\frac{k}{\sqrt{h}}\right) .
$$

Since $c_{1} k \leq h \leq c_{2} k$, we obtain

$$
\left|u(n h, x)-u_{k}^{h}(n, x)\right| \leq C \sqrt{k} .
$$

\subsubsection{Definitions and auxiliary properties}

Let $\beta(\cdot) \in C^{\infty}\left(\mathbb{R}^{r}\right)$, with the following properties

$$
\mid \begin{aligned}
& \beta(x) \geq 0, \quad \forall x \in \mathbb{R}^{r}, \\
& \operatorname{supp}(\beta) \subseteq B(0,1), \\
& \int_{\mathbb{R}^{r}} \beta(x) d x=1,
\end{aligned}
$$

where $\operatorname{supp}(\beta)=\left\{x \in \mathbb{R}^{r}: \beta(x) \neq 0\right\}$.

We define, the function $u_{\rho}^{h}$, regularization of the function $u^{h}$, as the convolution between $u^{h}$ and $\beta_{\rho}$, i.e.

$$
u_{\rho}^{h}(n, x)=\int_{\mathbb{R}^{r}} u^{h}(n, x-y) \beta_{\rho}(y) d y,
$$

where

$$
\beta_{\rho}(x)=\rho^{-r} \beta(x / \rho), \quad \forall \rho \in \mathbb{R}^{+} .
$$

By virtue of the convolution properties, $\forall n=0, \ldots, \mu, \forall x, \widehat{x} \in \mathbb{R}^{r}$, the function $u_{\rho}^{h}$ verifies

$$
\begin{gathered}
\left|u_{\rho}^{h}(n, x)-u_{\rho}^{h}(n, \widehat{x})\right| \leq L_{u}\|x-\widehat{x}\|, \\
\left|u_{\rho}^{h}(n, x)-u^{h}(n, x)\right| \leq L_{u} \rho .
\end{gathered}
$$

In the following lemmas we present auxiliary results which enable us to prove Theorem 3.1. Their proofs are given in the Appendix.

Lemma 3.1. For every $x \in V_{k}, n=0, \ldots, \mu-1$, it results

$$
u_{\rho}^{h}(n, x) \leq \min _{a \in A}\left\{\max \left\{f_{\rho}(x, a), u_{\rho}^{h}(n+1, x+h g(x, a))+L_{u} L_{g} h \rho\right\}\right\},
$$


where

$$
f_{\rho}(x, a)=f(x, a)+K \rho \text { and } K=L_{f}+L_{u}\left(2+L_{g} h\right) .
$$

From the previous lemma, we get the following corollary which establishes that the function $u_{\rho}^{h}$ is a subsolution of the perturbed problem (58), or in other words, that the inequality (59) holds.

Corollary 3.1. Let $v_{\rho}$ be the element of $C\left(\left(\mathbb{R}^{r}\right)^{\mu+1}\right)$ recursively defined by

$$
\mid \begin{array}{lrl}
v_{\rho}(n, x)=\min _{a \in A}\left\{\max \left\{f_{\rho}(x, a), v_{\rho}(n+1, x+h g(x, a))+L_{u} L_{g} h \rho\right\}\right\}, & \\
v_{\rho}(\mu, x)=\min _{a \in A} f_{\rho}(x, a) . & \forall n=0, \ldots, \mu-1,
\end{array}
$$

Then, $\forall x \in V_{k}, \forall n=0, \ldots, \mu$, it results

$$
u_{\rho}^{h}(n, x) \leq v_{\rho}(n, x) .
$$

Definition 3.1. We denote with $u_{\rho, k}^{h}$ the linear interpolation of $u_{\rho}^{h}$. In other words, the element of $F_{k}$, such that $\forall n=0, \ldots, \mu$, and $\forall x \in V_{k}$, it verifies

$$
u_{\rho, k}^{h}(n, x)=u_{\rho}^{h}(n, x) .
$$

In [23], it is proved that the difference between $u_{\rho}^{h}$ and its linear interpolation can be estimated in the following way:

$$
\left|u_{\rho, k}^{h}(n, x)-u_{\rho}^{h}(n, x)\right| \leq L_{u} \frac{k^{2}}{\rho} \quad \forall x \in \mathbb{R}^{r} .
$$

The following lemma establishes that the function $u_{\rho, k}^{h}$ is a subsolution of the problem (62).

Lemma 3.2. Let

$$
\begin{array}{lr}
\varepsilon_{\rho, k}(n, x)=\min _{a \in A}\left\{\max \left\{f_{\rho}(x, a), \varepsilon_{\rho, k}(n+1, x+h g(x, a))+L_{u} L_{g} h \rho+L_{u} \frac{k^{2}}{\rho}\right\}\right\}, \\
\varepsilon_{\rho, k}(\mu, x)=\min _{a \in A} f_{\rho}(x, a), & \forall n=0, \ldots, \mu-1,
\end{array}
$$

then, $\forall x \in V_{k}, \forall n \in\{0, \ldots, \mu\}$, it holds

$$
u_{\rho, k}^{h}(n, x) \leq \varepsilon_{\rho, k}(n, x) .
$$

In the lemma below we show a relation between the element $\varepsilon_{\rho, k}$ defined in (62) and the solution of the fully discrete problem.

Lemma 3.3. Let $x \in V_{k}$ and $n \in\{0, \ldots, \mu\}$, then

$$
\varepsilon_{\rho, k}(n, x) \leq u_{k}^{h}(n, x)+K \rho+T\left(L_{u} L_{g} \rho+L_{u} \frac{k^{2}}{h \rho}\right) .
$$


Definition 3.2. Let $\left\{a_{n}: n=0, \ldots, \mu\right\}$ be a sequence of elements of $A$. We define $z$ the function that satisfies

$$
\mid \begin{aligned}
& z(n, x)=\max \left\{f\left(x, a_{n}\right), z\left(n+1, x+h g\left(x, a_{n}\right)\right)\right\}, \quad \forall n=0, \ldots, \mu-1 . \\
& z(\mu, x)=f\left(x, a_{\mu}\right) .
\end{aligned}
$$

Remark 3.3. The function $z$ is the cost corresponding to the functional (2), where, although the control policy $\left\{a_{n}\right\}$ can be non-stationary, it does not depend on the state of the system. Clearly, $z$ depends on $\left\{a_{n}\right\}$; however, we do not explicitly write this dependence in order to simplify the notation. The function $z$ has properties similar to those of $u$, i.e.

$$
|z(n, x)-z(n, \widehat{x})| \leq L_{u}\|x-\widehat{x}\| .
$$

Definition 3.3. We denote with $z_{\rho}$ the regularized function of $z$, i.e. $\forall n=0, \ldots, \mu$

$$
z_{\rho}(n, x)=\int_{\mathbb{R}^{r}} z(n, x-y) \beta_{\rho}(y) d y,
$$

where $\beta_{\rho}$ is the function defined in (54).

Remark 3.4. By the properties of convolution, it is valid that $\forall n=0, \ldots, \mu, \forall x, \widehat{x} \in \mathbb{R}^{r}$, the function $z_{\rho}$ verifies

$$
\begin{gathered}
\left|z_{\rho}(n, x)-z_{\rho}(n, \widehat{x})\right| \leq L_{u}\|x-\widehat{x}\|, \\
\left|z_{\rho}(n, x)-z(n, x)\right| \leq L_{u} \rho .
\end{gathered}
$$

Definition 3.4. Let $z_{\rho, k}$ be the linear interpolation of $z_{\rho}$. In other words, the function of $F_{k}$ such that, $\forall x \in V_{k}, \forall n=0, \ldots, \mu$, verifies

$$
z_{\rho, k}(n, x)=z_{\rho}(n, x)
$$

We use again the result obtained in [23], which establishes that the difference between $z_{\rho}$ and its linear interpolation can be estimated, $\forall x \in \mathbb{R}^{r}$, in the following way

$$
\left|z_{\rho, k}(n, x)-z_{\rho}(n, x)\right| \leq L_{u} \frac{k^{2}}{\rho} .
$$

Lemma 3.4. Let $x \in V_{k}, n \in\{0, \ldots, \mu\}$ and let $\tilde{a}=\left\{\tilde{a}_{m}: m=0, \ldots, \mu\right\}$ be a control policy whose restriction for the values $n, n+1, \ldots, \mu$ is an optimal policy for the initial conditions $(n, x)$; i.e.

$$
J^{h}(n, x, \tilde{a})=u^{h}(n, x) .
$$

Then, $\forall m=n, \ldots, \mu$

$$
z\left(m, y_{x}^{h}(m)\right)=u^{h}\left(m, y_{x}^{h}(m)\right)
$$

where $y_{x}^{h}$ is defined by the recurrence

$$
\mid \begin{aligned}
& y_{x}^{h}(m+1)=y_{x}^{h}(m)+h g\left(y_{x}^{h}(m), \tilde{a}_{m}\right) \quad n \leq m \leq \mu-1, \\
& y_{x}^{h}(n)=x
\end{aligned}
$$


and $z$ is the function defined by (65), where $\left\{a_{m}\right\}$ is replaced by the special sequence $\left\{\tilde{a}_{m}\right\}$.

The following lemma establishes that the function $z_{\rho}$ is a supersolution of the problem associated to the inequality (71).

Lemma 3.5. For every $x \in V_{k}$ and $m=0, \ldots, \mu-1$, it results

$$
z_{\rho}(m, x) \geq \max \left\{\tilde{f}_{\rho}\left(x, \tilde{a}_{m}\right), z_{\rho}\left(m+1, x+h g\left(x, \tilde{a}_{m}\right)\right)-L_{u} L_{g} h \rho\right\}
$$

where

$$
\widetilde{f}_{\rho}\left(x, \tilde{a}_{m}\right)=f\left(x, \tilde{a}_{m}\right)-K \rho \text { and } K=L_{f}+L_{u}\left(2+L_{g} h\right) .
$$

Now, we show that the function $z_{\rho, k}$ given by Definition 3.4, is a supersolution of the problem (72) introduced below.

Lemma 3.6. Let $\sigma_{\rho, k}$ be the element of $F_{k}$ recursively defined by

$$
\mid \begin{array}{lr}
\sigma_{\rho, k}(n, x)=\max \left\{\tilde{f}_{\rho}\left(x, \tilde{a}_{n}\right), \sigma_{\rho, k}\left(n+1, x+h g\left(x, \tilde{a}_{n}\right)\right)-L_{u} L_{g} h \rho-L_{u} \frac{k^{2}}{\rho}\right\}, \\
\sigma_{\rho, k}(\mu, x)=\tilde{f}_{\rho}\left(x, \tilde{a}_{\mu}\right), & n=0, \ldots, \mu-1,
\end{array}
$$

then, $\forall x \in V_{k}, \forall m=0, \ldots, \mu$

$$
z_{\rho, k}(m, x) \geq \sigma_{\rho, k}(m, x) .
$$

The following lemma establishes that the solution of the fully discrete problem defined in (50) is a subsolution of the stopping time problem (74) (a maximizing problem), defined as follows.

Lemma 3.7. Let $\xi_{k}$ be the element of $F_{k}$ recursively defined by

$$
\mid \begin{aligned}
& \xi_{k}(m, x)=\max \left\{f\left(x, \tilde{a}_{m}\right), \xi_{k}\left(m+1, x+h g\left(x, \tilde{a}_{m}\right)\right)\right\}, m=0, \ldots, \mu-1, \\
& \xi_{k}(\mu, x)=f\left(x, \tilde{a}_{\mu}\right),
\end{aligned}
$$

then $\forall m=0, \ldots, \mu, \forall x \in V_{k}$, we have

$$
\xi_{k}(m, x) \geq u_{k}^{h}(m, x)
$$

In the lemma below we show a relation between the solution of problem (74) and the solution of problem (72).

Lemma 3.8. For every $x \in V_{k}, m=0, \ldots, \mu$, it results

$$
\sigma_{\rho, k}(m, x) \geq \xi_{k}(m, x)-K \rho-T\left(L_{u} L_{g} \rho+L_{u} \frac{k^{2}}{h \rho}\right) .
$$




\subsubsection{Proof of a preliminary estimate}

Now we can prove Theorem 3.1. To do that, we write

$$
\begin{aligned}
u^{h}(n, x)-u_{k}^{h}(n, x)= & \left(u^{h}(n, x)-u_{\rho}^{h}(n, x)\right)+\left(u_{\rho}^{h}(n, x)-u_{\rho, k}^{h}(n, x)\right) \\
& +\left(u_{\rho, k}^{h}(n, x)-\varepsilon_{\rho, k}(n, x)\right)+\left(\varepsilon_{\rho, k}(n, x)-u_{k}^{h}(n, x)\right) .
\end{aligned}
$$

The terms of (77) can be estimated in the following way:

By virtue of (56), we have

$$
u^{h}(n, x)-u_{\rho}^{h}(n, x) \leq L_{u} \rho,
$$

also, by $(60)$

$$
u_{\rho}^{h}(n, x)-u_{\rho, k}^{h}(n, x)=0,
$$

by Lemma 3.2

$$
u_{\rho, k}^{h}(n, x)-\varepsilon_{\rho, k}(n, x) \leq 0,
$$

and by Lemma 3.3

$$
\varepsilon_{\rho, k}(n, x)-u_{k}^{h}(n, x) \leq K \rho+T\left(L_{u} L_{g} \rho+L_{u} \frac{k^{2}}{h \rho}\right) .
$$

Therefore

$$
u^{h}(n, x)-u_{k}^{h}(n, x) \leq M \rho+T\left(L_{u} L_{g} \rho+L_{u} \frac{k^{2}}{h \rho}\right) .
$$

Let now $n$ and $x$ be arbitrary elements and $\tilde{a}$ be a control policy which restricted to the values $m=n, \ldots, \mu$, is a discrete optimal policy for the functional $J^{h}$ and the initial values $(n, x)$ (cf. Lemma 3.4). The function $z$ used in the remains of the proof is chosen according to this $\tilde{a}$. To obtain an inequality similar to (78) but where the sign $\geq$ holds, we write

$$
\begin{aligned}
u^{h}(n, x)-u_{k}^{h}(n, x)= & \left(u^{h}(n, x)-z(n, x)\right)+\left(z(n, x)-z_{\rho}(n, x)\right) \\
& +\left(z_{\rho}(n, x)-z_{\rho, k}(n, x)\right)+\left(z_{\rho, k}(n, x)-\sigma_{\rho, k}(n, x)\right) \\
& +\left(\sigma_{\rho, k}(n, x)-\xi_{k}(n, x)\right)+\left(\xi_{k}(n, x)-u_{k}^{h}(n, x)\right) .
\end{aligned}
$$

These terms are estimated in the following form:

By Lemma 3.4

$$
u^{h}(n, x)-z(n, x)=0,
$$


by $(67)$

$$
z(n, x)-z_{\rho}(n, x) \geq-L_{u} \rho
$$

by $(68)$

$$
z_{\rho}(n, x)-z_{\rho, k}(n, x)=0
$$

by Lemma 3.6

$$
z_{\rho, k}(n, x)-\sigma_{\rho, k}(n, x) \geq 0
$$

by Lemma 3.8

$$
\sigma_{\rho, k}(n, x)-\xi_{k}(n, x) \geq-K \rho-T\left(L_{u} L_{g} \rho+L_{u} \frac{k^{2}}{h \rho}\right)
$$

and, by Lemma 3.7

$$
\xi_{k}(n, x)-u_{k}^{h}(n, x) \geq 0
$$

In consequence,

$$
u^{h}(n, x)-u_{k}^{h}(n, x) \geq-M \rho-T\left(L_{u} L_{g} \rho+L_{u} \frac{k^{2}}{h \rho}\right) .
$$

From $(78,80)$, we obtain the inequality $(51)$, i.e.

$$
\left|u^{h}(n, x)-u_{k}^{h}(n, x)\right| \leq M \rho+T\left(L_{u} L_{g} \rho+L_{u} \frac{k^{2}}{h \rho}\right) .
$$

\subsection{Optimality of the estimate}

In the minimax problem, even though the data $f$ and $g$ are semiconcave in $x$, it is not possible to improve the estimate $\sqrt{h}$ which appears in (41) - as it was done in the problem studied in [20,22]. In those papers, under semiconcavity hypothesis on $f$ and $g$, it was shown that the optimal cost function $u$ also results semiconcave. In that case, the estimate for $\left\|u-u^{h}\right\|$ can be improved to order $h$, improvement that was crucial to prove an estimate of type $k^{2 / 3}$ for the fully discrete approximation. The following example shows that, for the minimax problem, an improvement of this type cannot be expected.

Definition 3.5. We say that the function $q: \mathbb{R}^{m} \mapsto \mathbb{R}^{r}$ is semiconcave in $x$ iff there exists $C>0$ such that

$$
\|q(x+z)+q(x-z)-2 q(x)\| \leq C\|z\|^{2} .
$$

Example 3.1. An example where the optimal cost is not semiconcave despite the fact that the data are semiconcave. 


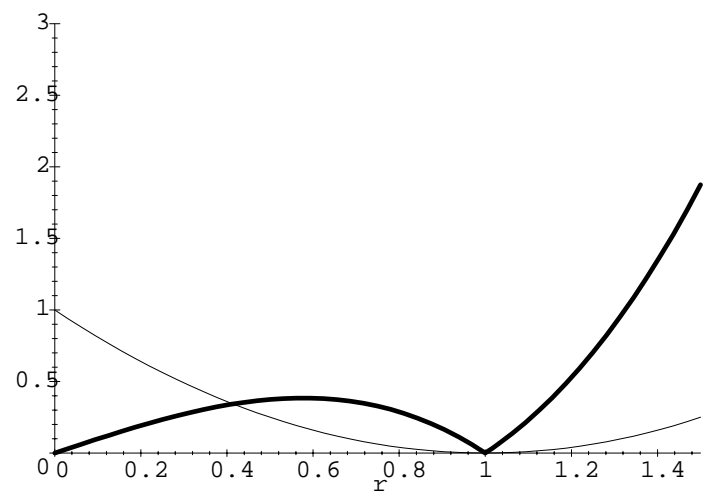

Figure 1. Optimal cost not semiconcave.

Let us consider a dynamic system which evolves in $\mathbb{R}^{2}$ according to the following ordinary differential equation

$$
\mid \begin{array}{ll}
y_{1}^{\prime}(s) & =y_{2}(s) \Psi(r(s)) \\
y_{2}^{\prime}(s) & =-y_{1}(s) \Psi(r(s)), \quad \forall s \in[0,10], \\
\left(y_{1}(0), y_{2}(0)\right) & =\left(x_{1}, x_{2}\right) \in \mathbb{R}^{2},
\end{array}
$$

and the instantaneous cost

$$
f\left(y_{1}(s), y_{2}(s)\right)=\left(1-\left(y_{1}^{2}(s)+y_{2}^{2}(s)\right)\right) y_{1}(s) \Psi(r(s)) .
$$

where $r(\cdot)=\sqrt{y_{1}^{2}(\cdot)+y_{2}^{2}(\cdot)}$ and $\Psi$ is given by

$$
\Psi(r)=\mid \begin{array}{ll}
1 & \text { if } r \leq 2 \\
e^{-(r-2)^{2}} & \text { if } r>2 .
\end{array}
$$

The system moves without restrictions in $\mathbb{R}^{2}$. The functions $f$ and $g$ verify the assumptions $A_{1}-A_{2}$ and clearly, both of them are semiconcave.

Let $r=r(0)=\sqrt{x_{1}^{2}+x_{2}^{2}}$. It is easy to check that the value function is $($ for $r \leq 2)$

$$
u\left(0, x_{1}, x_{2}\right)=\max _{s \in[0,10]} f\left(y_{1}(s), y_{2}(s)\right)=\left|1-r^{2}\right| r,
$$

for $r \in[0,2]$. The optimal cost for this problem is not semiconcave at $r=1$, as it can be seen in Figure 1 (also it is not semiconcave at $r=0$ ).

Moreover, the discretization procedure introduced here coincides with the methodology studied in [21]. In that work, the authors proved that the estimate $\sqrt{k}$ is optimal when $L_{g}<\lambda$, being $\lambda$ the discount factor of the cumulative problem. In fact, for the data of this example, we can prove - using the triangulation shown in Figure 2 and calculations entirely similar to those employed in $[21,22]$ - that the error $\left|u(0, x)-u_{k}^{h}(0, x)\right|$ verifies

$$
\left|u(0, x)-u_{k}^{h}(0, x)\right| \geq C \sqrt{k} .
$$




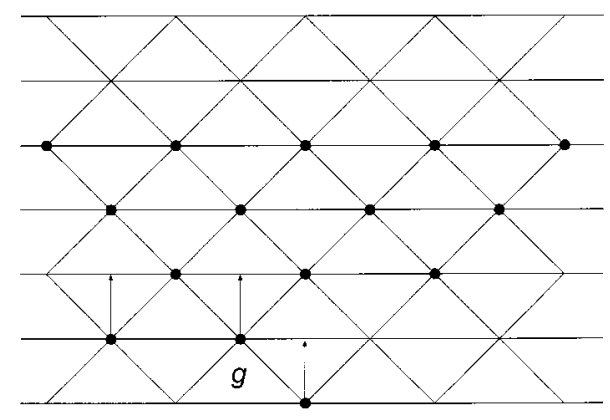

$(0,0)$

Figure 2. Mesh in $\mathbb{R}^{2}$.

Example 3.2. An example where the error of approximation is exactly of order $\sqrt{k}$.

We consider a dynamic system which evolves in $\mathbb{R}^{2}$ according to

$$
\mid \begin{aligned}
& \frac{d y}{d t}(s)=(0,1) \quad 0 \leq t \leq s \leq T, \\
& y(t)=\left(x_{1}, x_{2}\right) \quad\left(x_{1}, x_{2}\right) \in \mathbb{R}^{2} .
\end{aligned}
$$

The instantaneous cost $f$ is

$$
f\left(y_{1}(s), y_{2}(s), a\right)=a y_{1}(s)=a x_{1}, \quad a \in A=\{-1,1\} .
$$

It is easy to check that the value function results

$$
u\left(t, x_{1}, x_{2}\right)=\left|x_{1}\right| .
$$

In particular, for $\left(x_{1}, x_{2}\right)=(0,0)$ we have $u(t, 0,0)=0$.

We use here the triangulation shown in Figure 2. Evaluating (50) in this problem, it follows that

$$
u_{k}^{h}(0,0,0) \geq \frac{1}{2^{\mu}} \sum_{j=0}^{\mu}\left(\begin{array}{c}
\mu \\
j
\end{array}\right)\left|j-\frac{\mu}{2}\right| k
$$

where $\mu=T / h$.

By elementary calculations we have that

$$
u_{k}^{h}(0,0,0) \geq C \frac{\sqrt{\mu}}{2} k
$$

Taking $T=1$ and $h=k$, it results

$$
u_{k}^{h}-u \geq C \frac{\sqrt{k}}{2} .
$$

In consequence, for approximations of this type, the best error that can be expected is of order $\sqrt{k}$. 
Remark 3.5. The optimality of the estimate (85) stems from the fact that - as it was explained in Section 1 - our minimax problem is a disguised differential game problem. In that game, one controller tries to minimize the cost

$$
J(t, x, \alpha(\cdot), \tau)=f(y(\tau), \alpha(\tau))
$$

while the opponent - using full information of actions of the first player - chooses at any instant the stopping time $\tau$ of the process. As a result of the complete game, the pay-off (82) is given. As a consequence of the second player's privileged action, the first one must - in a strict way - minimize a functional that is not semiconcave with respect to the spatial variable $y$. In this way, once a full discretization - using finite differences or finite elements - is applied, the resultant fully discrete optimal control problem reflects this property in the validity of the estimate of type $\sqrt{k}$.

Remark 3.6. It is easy to check that exactly the same results of convergence can be obtained if $f$ and $g$ depend on time and verify conditions similar to $\left(\mathrm{A}_{1}-\mathrm{A}_{3}\right)$. In this paper we have not considered this time dependence in order to simplify the exposition.

\section{NumericAl EXAMPLE}

The problem consists in the calculus of the optimal trajectory from a given initial position $x \in \mathbb{R}^{2}$ and the initial time in $[0, T]$. The functional $J$ to be minimized is given by

$$
J(t, x, a)=\max _{s \in[t, T]} f(s, y(s)),
$$

where $y(\cdot)$ is the trajectory of the system. The function $f:[0, T] \times \mathbb{R}^{2} \mapsto \mathbb{R}$ is independent on the control and has the shape shown in Figure 3 (this figure corresponds to the function $f(0, \cdot)$ ). The optimal trajectory minimizes the maximum value of $f$ along its way.

In this problem, $A=\{0,1,2,3,4\}$ and the dynamic $g$ defined below gives five admissible directions of movement on $\mathbb{R}^{2}$

$$
g(x, a)=\min \left(1, \frac{d(x, \partial D)}{2}\right) \times \chi(D) \times \mid \begin{array}{ll}
(0,0) & \text { if } a=0 \\
(1,0) & \text { if } a=1 \\
(0,1) & \text { if } a=2 \\
(-1,0) & \text { if } a=3 \\
(0,-1) & \text { if } a=4
\end{array}
$$

Here, $D$ is the rectangular domain $D=[-4,4] \times[-4,4]$ and $\chi(D)$ is the characteristic function of $D$.

We consider a regular discretization of $\mathbb{R}^{2}$, whose nodes have the general form

$$
(i k, j k), \quad i \in Z, \quad j \in Z \text {. }
$$

We divide the time interval $[0, T]$ into $\mu$ sub-intervals with common length $h=T / \mu$.

In consequence, (50) becomes, $\forall i, j$

$$
\mid \begin{array}{lr}
\begin{array}{ll}
u_{k}^{h}(n, i k, j k)=\max \left\{f(n h, i k, j k), \min _{a \in A} u_{k}^{h}(n+1,(i k, j k)\right. & +h g(i k, j k, a))\}, \\
u_{k}^{h}(\mu, i k, j k)=f(\mu h, i k, j k) . & \forall n=0, \ldots, \mu-1,
\end{array}
\end{array}
$$




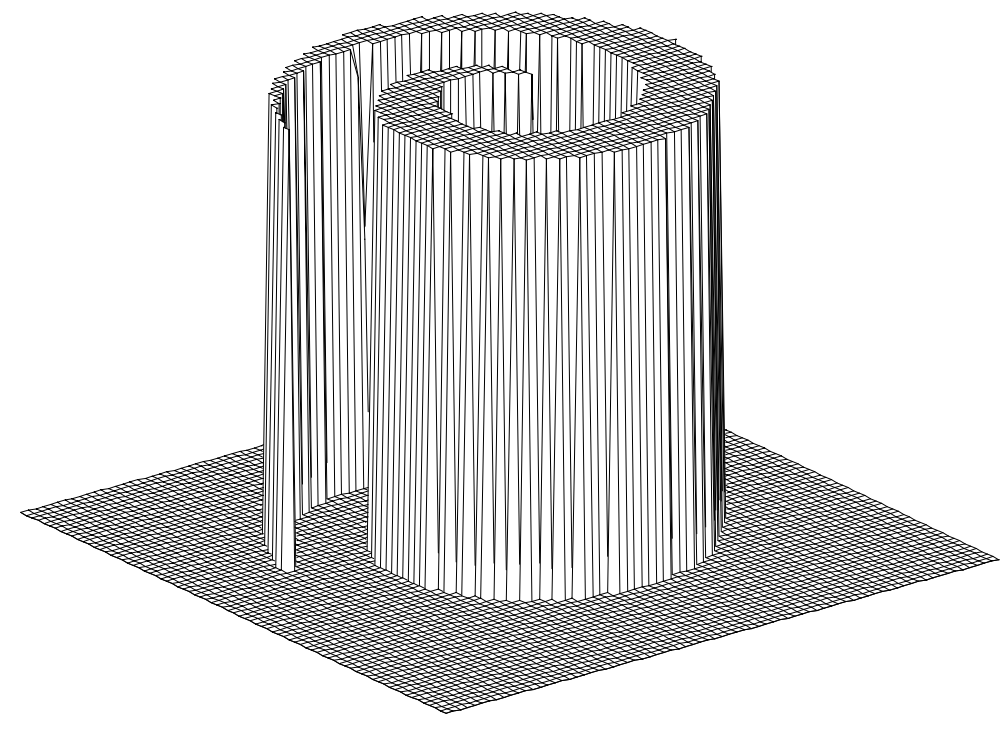

Figure 3. Function shape.

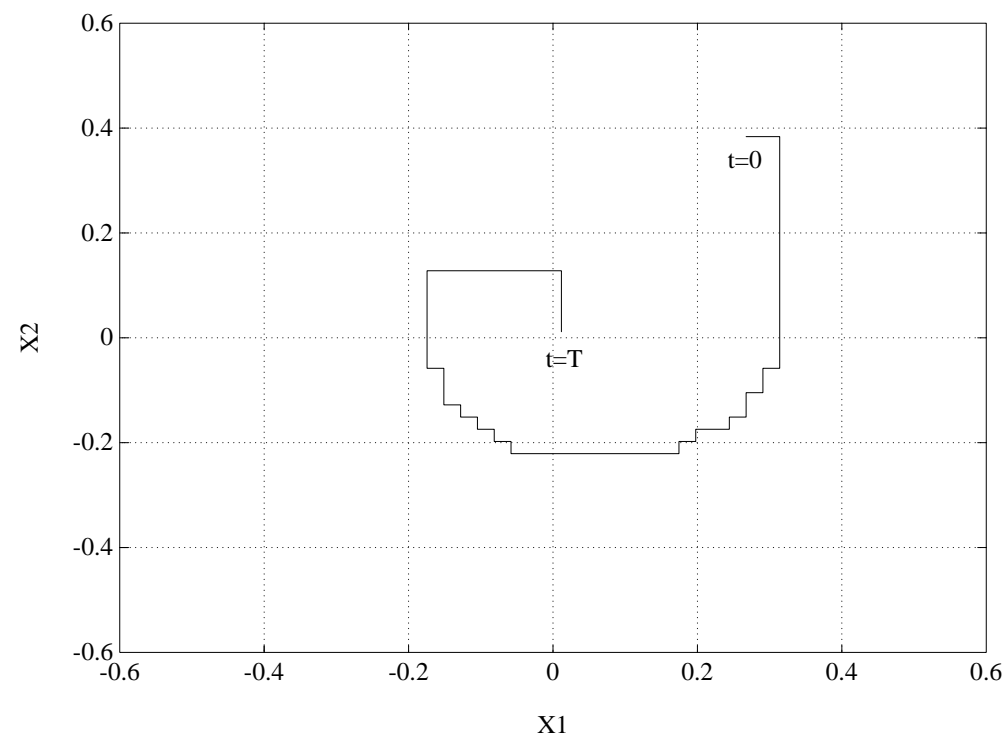

Figure 4. Optimal trajectory.

This system gives a recursive procedure to compute the optimal cost at each time and at each node. At each iteration, the direction that produces the optimum is stored, operation which enables us to construct the optimal trajectories, like that one shown in Figure 3.

In the particular case where the points belong to the square $D$, all computations can be carried out using only the values of $u_{k}^{h}$ at the points $\left(n h, x_{i}\right), x_{i} \in D n=0, \ldots, \mu$, because for those points appearing at the left hand side of equation (83), the corresponding points appearing at the right hand side are also points of the form $\left(n h, x_{i}\right), x_{i} \in D n=0, \ldots, \mu$. We have profited of this property to obtain the optimal trajectory shown in Figure 4. 


\section{Generalizations}

The previous procedure can be extended to the case where the functional includes a final cost. More specifically, we consider the case where the functional to be minimized takes the form

$$
\bar{J}(t, x, \alpha(\cdot))=\max \{J(t, x, \alpha(\cdot)), \Psi(y(T))\} .
$$

In this case, the optimal cost verifies a dynamic programming principle of the following form: $\forall t \in[0, T)$, $\forall s \in[0, T] s>t, \forall x \in \mathbb{R}^{r}$

$$
\begin{aligned}
& u(t, x)=\inf _{\alpha \in \mathcal{A}_{t}}\{\max \{u(s, y(s)), \underset{\tau \in[t, s]}{\operatorname{ess} \sup } f(y(\tau), \alpha(\tau))\}\}, \\
& u(T, x)=\max \left\{\min _{a \in A} f(x, a), \Psi(x)\right\} .
\end{aligned}
$$

The fully discrete solution verifies the following recurrence $\forall x^{i} \in V_{k}$

$$
\mid \begin{aligned}
& u_{k}^{h}\left(n, x^{i}\right)=\min _{a \in A}\left\{\max \left\{f\left(x^{i}, a\right), \sum_{j=1}^{\infty} \gamma_{j}\left(x^{i}, a\right) u_{k}^{h}\left(n+1, x^{j}\right)\right\}\right\}, \\
& u_{k}^{h}\left(\mu, x^{i}\right)=\max \left\{\min _{a \in A} f\left(x^{i}, a\right), \Psi\left(x^{i}\right)\right\} .
\end{aligned}
$$

\section{Conclusions}

Here, we have developed a discretization procedure to obtain the numerical solution of the problem of minimizing the maximum cost, analyzed from the continuous point of view by Barron-Ishii in [5].

The numerical procedure obtained is easily implementable and it converges to the solution of the original problem, with an error estimate of the form

$$
\left|u-u_{k}^{h}\right| \leq C \sqrt{k}
$$

This estimate was shown to be optimal.

Except for very special trajectories, where some carefully chosen triangulations may be used, it seems not possible in general to get approximations with better convergence properties.

\section{APPENDiX}

In this section, we give the proofs corresponding to the lemmas introduced in Section 3.

Proof of Lemma 3.1. Let $x \in V_{k}$ and $n=0, \ldots, \mu-1$; as we have proved in Lemma 2.1,

$$
\mid \begin{aligned}
& u^{h}(n, x)=\min _{a \in A}\left\{\max \left\{f(x, a), u^{h}(n+1, x+h g(x, a))\right\}\right\}, \\
& u^{h}(\mu, x)=\min _{a \in A} f(x, a),
\end{aligned}
$$


then, $\forall a \in A, u^{h}$ satisfies

$$
u^{h}(n, x) \leq \max \left\{f(x, a), u^{h}(n+1, x+h g(x, a))\right\} .
$$

Let $R=L_{f}+L_{u}\left(1+L_{g} h\right)$.

We analyze two cases concerning $\max \left\{f(x, a), u^{h}(n+1, x+h g(x, a))\right\}$

1. $\max \left\{f(x, a), u^{h}(n+1, x+h g(x, a))\right\} \leq f(x, a)+R \rho$.

It results from $(86)$

$$
u^{h}(n, x) \leq f(x, a)+R \rho
$$

By using (56), we have

$$
u_{\rho}^{h}(n, x) \leq u^{h}(n, x)+L_{u} \rho
$$

consequently, from (87), it follows that

$$
u^{h}(n, x)+L_{u} \rho \leq f(x, a)+R \rho+L_{u} \rho=f(x, a)+K \rho=f_{\rho}(x, a) .
$$

From $(88,89)$, we obtain

$$
u_{\rho}^{h}(n, x) \leq f_{\rho}(x, a) .
$$

2. $\max \left\{f(x, a), u^{h}(n+1, x+h g(x, a))\right\}>f(x, a)+R \rho$.

This condition implies

$$
\max \left\{f(x, a), u^{h}(n+1, x+h g(x, a))\right\}=u^{h}(n+1, x+h g(x, a)) .
$$

Let us see that $\forall y \in B_{\rho}(x)$ the following inequality holds (where $B_{\rho}(x)$ denotes a ball centered on $x$ of radius $\rho$ ),

$$
u^{h}(n, y) \leq u^{h}(n+1, y+h g(y, a))
$$

Let us suppose that (92) is false, i.e., $\exists \tilde{y} \in B_{\rho}(x)$, such that

$$
u^{h}(n, \tilde{y})>u^{h}(n+1, \tilde{y}+h g(\tilde{y}, a)) .
$$

As $u^{h}(n, \tilde{y}) \leq \max \left\{f(\tilde{y}, a), u^{h}(n+1, \tilde{y}+h g(\tilde{y}, a))\right\}$, the inequality (93) implies

$$
f(\tilde{y}, a)>u^{h}(n+1, \tilde{y}+h g(\tilde{y}, a)) .
$$

By virtue of the fact that $f$ and $u^{h}$ are Lipschitz continuous, we obtain

$$
\begin{aligned}
f(x, a)+L_{f} \rho & \geq f(\tilde{y}, a)>u^{h}(n+1, \tilde{y}+h g(\tilde{y}, a)) \\
& \geq u^{h}(n+1, x+h g(x, a))-L_{u}\left(1+L_{g} h\right) \rho .
\end{aligned}
$$


Hence,

$$
u^{h}(n+1, x+h g(x, a))-f(x, a)<L_{f} \rho+L_{u}\left(1+L_{g} h\right) \rho=R \rho,
$$

which contradicts (91).

By convolution of $u^{h}$ with $\beta_{\rho}$ we have, by virtue of (92),

$$
\begin{array}{r}
u_{\rho}^{h}(n, x)=\int_{B_{\rho}(x)} u^{h}(n, x-y) \beta_{\rho}(y) d y \\
\leq \int_{B_{\rho}(x)} u^{h}(n+1, x-y+h g(x-y, a)) \beta_{\rho}(y) d y \\
\leq \int_{B_{\rho}(x)} u^{h}(n+1, x-y+h g(x, a)) \beta_{\rho}(y) d y \\
+\int_{B_{\rho}(x)} L_{u}\|x-y+h g(x-y, a)-(x-y+h g(x, a))\| \beta_{\rho}(y) d y \\
\leq u_{\rho}^{h}(n+1, x+h g(x, a))+L_{u} L_{g} h \int_{B_{\rho}(x)} \beta_{\rho}(y) d y \\
=u_{\rho}^{h}(n+1, x+h g(x, a))+L_{u} L_{g} h \rho .
\end{array}
$$

Consequently,

$$
u_{\rho}^{h}(n, x) \leq u_{\rho}^{h}(n+1, x+h g(x, a))+L_{u} L_{g} h \rho .
$$

From $(90,94)$, it follows that

$$
u_{\rho}^{h}(n, x) \leq \min _{a \in A}\left\{\max \left\{f_{\rho}(x, a), u_{\rho}^{h}(n+1, x+h g(x, a))+L_{u} L_{g} h \rho\right\}\right\} .
$$

Proof of Corollary 3.1. For $n=\mu$, the following relation holds

$$
u_{\rho}^{h}(\mu, x) \leq u^{h}(\mu, x)+L_{u} \rho=\min _{a \in A} f(x, a)+L_{u} \rho \leq \min _{a \in A} f_{\rho}(x, a)=v_{\rho}(\mu, x) .
$$

From $(58,95,96)$, we can prove by induction the following inequality

$$
u_{\rho}^{h} \leq v_{\rho} .
$$

From (96), the relation (97) holds for $n=\mu$. Let us assume that it is true for $n=m+1$ and we will prove that the same happens for $n=m$. From (95), the induction assumptions and (58), we have

$$
\begin{aligned}
u_{\rho}^{h}(m, x) & \leq \min _{a \in A}\left\{\max \left\{f_{\rho}(x, a), u_{\rho}^{h}(m+1, x+h g(x, a))+L_{u} L_{g} h \rho\right\}\right\} \\
& \leq \min _{a \in A}\left\{\max \left\{f_{\rho}(x, a), v_{\rho}(m+1, x+h g(x, a))+L_{u} L_{g} h \rho\right\}\right\}=v_{\rho}(m, x) .
\end{aligned}
$$


Proof of Lemma 3.2. From the definition of $u_{\rho}^{h}$ and (60), we have

$$
u_{\rho, k}^{h}(\mu, x)=u_{\rho}^{h}(\mu, x) \leq \varepsilon_{\rho, k}(\mu, x)
$$

Let $x \in V_{k}$ and $n=0, \ldots, \mu-1$; from Lemma 3.1, it follows that, $\forall a \in A$,

$$
u_{\rho}^{h}(n, x) \leq \max \left\{f_{\rho}(x, a), u_{\rho}^{h}(n+1, x+h g(x, a))+L_{u} L_{g} h \rho\right\} .
$$

We analyze two cases concerning $\max \left\{f_{\rho}(x, a), u_{\rho}^{h}(n+1, x+h g(x, a))+L_{u} L_{g} h \rho\right\}$

1. $\max \left\{f_{\rho}(x, a), u_{\rho}^{h}(n+1, x+h g(x, a))+L_{u} L_{g} h \rho\right\}=f_{\rho}(x, a)$.

In this case (60) implies

$$
u_{\rho, k}^{h}(n, x)=u_{\rho}^{h}(n, x) \leq f_{\rho}(x, a)
$$

2. $\max \left\{f_{\rho}(x, a), u_{\rho}^{h}(n+1, x+h g(x, a))+L_{u} L_{g} h \rho\right\}=u_{\rho}^{h}(n+1, x+h g(x, a))+L_{u} L_{g} h \rho$.

Here, from (60), it results

$$
u_{\rho, k}^{h}(n, x)=u_{\rho}^{h}(n, x) \leq u_{\rho}^{h}(n+1, x+h g(x, a))+L_{u} L_{g} h \rho .
$$

Using (61), we obtain

$$
u_{\rho, k}^{h}(n, x) \leq u_{\rho, k}^{h}(n+1, x+h g(x, a))+L_{u} L_{g} h \rho+L_{u} \frac{k^{2}}{\rho} .
$$

For each $a \in A$, at least one of the inequalities (99) or (100), must be true. Consequently,

$$
u_{\rho, k}^{h}(n, x) \leq \max \left\{f_{\rho}(x, a), u_{\rho, k}^{h}(n+1, x+h g(x, a))+L_{u} L_{g} h \rho+L_{u} \frac{k^{2}}{\rho}\right\},
$$

then, since $a$ is an arbitrary element of $A$, we have

$$
u_{\rho, k}^{h}(n, x) \leq \min _{a \in A}\left\{\max \left\{f_{\rho}(x, a), u_{\rho, k}^{h}(n+1, x+h g(x, a))+L_{u} L_{g} h \rho+L_{u} \frac{k^{2}}{\rho}\right\}\right\} .
$$

From $(98,101)$, also by induction, we can prove as we have done in Corollary 3.1 that $u_{\rho, k}^{h} \leq \varepsilon_{\rho, k}$.

Proof of Lemma 3.3. From (50), we have $u_{k}^{h}(\mu, x)=\min _{a \in A} f(x, a)$. By definition of $\varepsilon_{\rho, k}$, we obtain

$$
\varepsilon_{\rho, k}(\mu, x)=\min _{a \in A} f_{\rho}(x, a)=\min _{a \in A} f(x, a)+K \rho=u_{k}^{h}(\mu, x)+K \rho .
$$

Let us suppose that for a generic $n$,

$$
\varepsilon_{\rho, k}(n, x) \leq u_{k}^{h}(n, x)+K \rho+(\mu-n)\left(L_{u} L_{g} h \rho+L_{u} \frac{k^{2}}{\rho}\right)
$$

The previous inequality also holds in points of the form $x+h g(x, a)$ that do not necessarily belong to $V_{k}$. This is because the functions $u_{k}^{h}$ and $\varepsilon_{\rho, k}$ are in $F_{k}$, so they verify the property of linearity and the assumption (49). 
We must prove the inequality for $n-1$

$$
\begin{aligned}
& \varepsilon_{\rho, k}(n-1, x)=\min _{a \in A}\left\{\max \left\{f_{\rho}(x, a), \varepsilon_{\rho, k}(n, x+h g(x, a))+L_{u} L_{g} h \rho+L_{u} \frac{k^{2}}{\rho}\right\}\right\} \\
& \leq \min _{a \in A}\left\{\max \left\{f_{\rho}(x, a), u_{k}^{h}(n, x+h g(x, a))+K \rho+(\mu-n+1)\left(L_{u} L_{g} h \rho+L_{u} \frac{k^{2}}{\rho}\right)\right\}\right\} \\
& \leq \min _{a \in A}\left\{\max \left\{f_{\rho}(x, a), u_{k}^{h}(n, x+h g(x, a))\right\}\right\}+K \rho+(\mu-n+1)\left(L_{u} L_{g} h \rho+L_{u} \frac{k^{2}}{\rho}\right) \\
& =u_{k}^{h}(n-1, x)+K \rho+(\mu-n+1)\left(L_{u} L_{g} h \rho+L_{u} \frac{k^{2}}{\rho}\right) .
\end{aligned}
$$

Consequently, by induction,

$$
\begin{aligned}
\varepsilon_{\rho, k}(0, x) & \leq u_{k}^{h}(0, x)+K \rho+\mu\left(L_{u} L_{g} h \rho+L_{u} \frac{k^{2}}{\rho}\right) \\
& \leq u_{k}^{h}(0, x)+K \rho+T\left(L_{u} L_{g} \rho+L_{u} \frac{k^{2}}{\rho h}\right) .
\end{aligned}
$$

Proof of Lemma 3.4. By definition of $\left\{\tilde{a}_{m}\right\}$ and by the dynamic programming principle, we have that

$$
\mid \begin{aligned}
& u^{h}\left(m, y_{x_{o}}^{h}(m)\right)=\max \left\{f\left(y_{x_{o}}^{h}(m), \tilde{a}_{m}\right), u^{h}\left(m+1, y_{x_{o}}^{h}(m)+h g\left(y_{x_{o}}^{h}(m), \tilde{a}_{m}\right)\right\}\right. \\
& u^{h}\left(\mu, y_{x_{o}}^{h}(\mu)\right)=f\left(y_{x_{o}}^{h}(\mu), \tilde{a}_{\mu}\right) .
\end{aligned}
$$

Then, $\forall m=n, \ldots, \mu$, it follows that $z\left(n, y_{x_{o}}^{h}(n)\right)=u^{h}\left(n, y_{x_{o}}^{h}(n)\right)$.

Proof of Lemma 3.5. It is similar to the proof of Lemma 3.1, and it is here omitted.

Proof of Lemma 3.6. Let $x \in V_{k}$ and $n \in\{0, \ldots, \mu-1\}$ (the proof is obvious for $n=\mu$ ). According to the definition of $z_{\rho, k}$ and the result of Lemma 3.5, the following relations are valid:

$$
\begin{gathered}
z_{\rho, k}(n, x)=z_{\rho}(n, x) \geq \tilde{f}_{\rho}\left(x, \tilde{a}_{n}\right), \\
z_{\rho, k}(n, x)=z_{\rho}(n, x) \geq z_{\rho}\left(n+1, x+h g\left(x, \tilde{a}_{n}\right)\right)-L_{u} L_{g} h \rho .
\end{gathered}
$$

Using (69), we obtain

$$
z_{\rho}\left(n+1, x+h g\left(x, \tilde{a}_{n}\right)\right) \geq z_{\rho, k}\left(n+1, x+h g\left(x, \tilde{a}_{n}\right)\right)-L_{u} \frac{k^{2}}{\rho} .
$$

From $(103,104)$ we arrive to

$$
z_{\rho, k}(n, x) \geq z_{\rho, k}\left(n+1, x+h g\left(x, \tilde{a}_{n}\right)\right)-L_{u} L_{g} h \rho-L_{u} \frac{k^{2}}{\rho} .
$$


Therefore, from $(102,105), z_{\rho, k}$ verifies

$$
z_{\rho, k}(n, x) \geq \max \left\{\tilde{f}\left(x, \tilde{a}_{n}\right), z_{\rho, k}\left(n+1, x+h g\left(x, \tilde{a}_{n}\right)\right)-L_{u} L_{g} h \rho-L_{u} \frac{k^{2}}{\rho}\right\} .
$$

The inequality (73) can be proved by induction as we have done in Lemma 3.1 because the relation for the final condition is obviously true.

Proof of Lemma 3.7. Taking in mind the definition of $\xi_{k}$, we obtain

$$
\xi_{k}(\mu, x)=f\left(x, \tilde{a}_{\mu}\right) \geq \min _{a \in A} f(x, a)=u_{k}^{h}(\mu, x) .
$$

Given $m \in\{0, \ldots, \mu-1\}$, let us suppose that, $\forall x \in V_{k}$,

$$
\xi_{k}(m+1, x) \geq u_{k}^{h}(m+1, x) .
$$

As we have seen in the proof of Lemma 3.3, this inequality also holds at any point of the form $x+h g(x, a)$. Let us see that the inequality (107) also holds for $m$.

$$
\begin{aligned}
\xi_{k}(m, x) & =\max \left\{f\left(x, \tilde{a}_{m}\right), \xi_{k}\left(m+1, x+h g\left(x, \tilde{a}_{m}\right)\right)\right\} \\
& \geq \max \left\{f\left(x, \tilde{a}_{m}\right), u_{k}^{h}\left(m+1, x+h g\left(x, \tilde{a}_{m}\right)\right)\right\} \\
& \geq \min _{a \in A}\left\{\max \left\{f(x, a), u_{k}^{h}(m+1, x+h g(x, a))\right\}\right\}=u_{k}^{h}(m, x) .
\end{aligned}
$$

Consequently, by induction we have, $\forall m=0, \ldots, \mu, \xi_{k}(m, x) \geq u_{k}^{h}(m, x)$.

Proof of Lemma 3.8. It is similar to the proof of Lemma 3.3 and it is here omitted.

\section{REFERENCES}

[1] J.P. Aubin and A. Cellina, Differential inclusions. Springer-Verlag, New York (1984).

[2] G. Barles, Ch. Daher and M. Romano, Optimal control on the $L^{\infty}$ norm of a diffusion process. SIAM J. Contr. Opt. 32 (1994) 612-634.

[3] G. Barles, Ch. Daher and M. Romano, Convergence of numerical schemes for parabolic equations arising in finance theory. Math. Models Met. Appl. Sci. 5 (1995) 125-143.

[4] E.N. Barron, Differential games with maximum cost. Nonlinear Analysis, Theory, Methods and Applications 14 (1990) $971-989$.

[5] E.N. Barron, The Pontryagin maximum principle for minimax problems of optimal control. Nonlinear Analysis, Theory, Methods and Applications 15 (1990) 1155-1165.

[6] E.N. Barron, Averaging in Lagrange and minimax problems of optimal control. SIAM J. Contr. Opt. 31 (1993) $1630-1652$.

[7] E.N. Barron, Optimal control and calculus of variations in $L^{\infty}$, in Optimal Control in Differential Equations. N.H. Pavel and Marcel Dekker Eds., New York (1994).

[8] E.N. Barron and H. Ishii, The Bellman equation for minimizing the maximum cost. Nonlinear Analysis, Theory, Methods and Applications 13 (1989) 1067-1090.

[9] E.N. Barron and R. Jensen, Relaxed minimax control. SIAM J. Contr. Opt. 33 (1995) 1028-1039.

[10] E.N. Barron, R. Jensen and J.L. Menaldi, Optimal control and differential games with measures. Nonlinear Analysis, Theory, Methods and Applications 21 (1993) 241-268.

[11] I. Capuzzo Dolcetta, On a discrete approximation of the Hamilton-Jacobi equation of dynamic programming. Appl. Math. Optim. 10 (1983) 367-377.

[12] I. Capuzzo Dolcetta and M. Falcone, Discrete dynamic programming and viscosity solutions of the Bellman equation. Ann. Inst. Henry Poincaré. Anal. Non-lin. 6 (1989) 161-184. 
[13] I. Capuzzo Dolcetta and H. Ishii, Approximate solution of the Bellman equation of deterministic control theory. Appl. Math. Optim. 11 (1984) 161-181.

[14] P.G. Ciarlet, Discrete maximum principle for finite-difference operators. Aequations Math. 4 (1970) 338-352.

[15] R. Dacorogna, Direct methods in the calculus of variations. Springer-Verlag, Berlin (1987).

[16] S.C. Di Marco and R.L.V. González, Une procédure numérique pour la minimisation du coût maximum. C. R. Acad. Sci. Paris, Série I 321 (1995) 869-874.

[17] S.C. Di Marco and R.L.V. González, A minimax optimal control problem wih infinite horizon. Rapport de Recherche $\mathrm{N}^{\circ} 2945$, INRIA, Rocquencourt (1996).

[18] A. Friedman, Differential games. Wiley-Interscience, New York (1971).

[19] R.L.V. González and E. Rofman, On deterministic control problems: An approximation procedure for the optimal cost, Parts 1 and 2, SIAM J. Contr. Opt. 23 (1985) 242-285.

[20] R.L.V. González and M.M. Tidball, On a discrete time approximation of the Hamilton-Jacobi equation of dynamic programming, Rapport de Recherche Nº1375, INRIA, Rocquencourt (1990).

[21] R.L.V. González and M.M. Tidball, On the rate of convergence of fully discrete solutions of Hamilton-Jacobi equations, Rapport de Recherche N¹376, INRIA, Rocquencourt (1991).

[22] R.L.V. González and M.M. Tidball, Sur l'ordre de convergence des solutions discrétisées en temps et en espace de l'équation de Hamilton-Jacobi, C. R. Acad. Sci., Paris, Série I 314 (1992) 479-482.

[23] G. Strang and G. Fix, An analysis of the finite element method. Prentice-Hall, Englewood Cliffs, New Jersey (1973). 